\title{
Boundary Condition Identification of a Plate on Elastic Support
}

\author{
Hamid Ahmadian and Morteza Esfandiar \\ Center of Excellence in Experimental Solid Mechanics and Dynamics, Iran University of Science and Technology, \\ Narmak, Tehran 16848, Iran
}

Hassan Jalali

Department of Mechanical Engineering, Arak University of Technology, Arak 38135-1177, Iran

(Received 29 December 2012; revised 27 March 2013; accepted: 26 February 2014)

\begin{abstract}
The behaviour of mechanical structures in low frequencies is strongly affected by the existence of the boundary conditions. It is not usually possible to provide ideal boundary conditions, i.e. simply supported or clamped, for structures. Therefore the real structures are mostly constrained by elastic supports. Constructing an accurate mathematical or numerical model for a structure requires the knowledge of the support parameters. In this paper, a new method is proposed for the parameter identification of a rectangular plate constrained by elastic support. The method relies on the free vibration solution of the plate dynamics subjected to elastic boundary conditions and employs the optimization toolbox of MATLAB.
\end{abstract}

\section{INTRODUCTION}

The supports, or boundary conditions, play an important role in a structure's dynamic behaviour and must be considered carefully when constructing mathematical or numerical models. In reality, the supports of structures are not rigid enough, and they show flexibility to some degree. The flexibility of the supports can be modelled as elastic boundary conditions. In order to have an accurate model of a structure, the knowledge of the support parameters is essential. The support parameters can be identified by using experimental results.

The sensitivity method is one of the most widely used approaches in determining boundary condition parameters. ${ }^{1}$ In this method the difference between model predictions and test observations is defined as an objective function. An iterative process is then adopted, and the objective function is minimized by using the sensitivity approach. It should be noted that the sensitivity of higher natural frequencies to support parameters is low, which results in convergence problems in the optimization procedure. $^{2}$

In the characteristic equation method the boundary support parameters are identified by solving the nonlinear characteristic equations. In this method, which was adopted by Ahmadian et al., the number of characteristic equations formed is equal to the number of measured natural frequencies. The boundary condition parameters are then identified by simultaneously solving the characteristic equations. ${ }^{3}$

Waters et al. and Wang and Yang adopted the static flexibility measurements and identified the boundary conditions of a tapered beam., ${ }^{4,5}$ They modelled the beam as a uniform rigid beam that was constrained by collocated equivalent trans- lational and rotational springs. The boundary conditions are identified by quasi-static stiffness measurements obtained from impact tests.

This paper deals with the support parameter identification of a rectangular plate constrained in its edges by an elastic boundary condition. The boundary condition contains structural damping. The solution method proposed by $\mathrm{Li}$ et al. is adopted to analyse the free vibration of the beam. ${ }^{6}$ The analysis leads to obtaining the natural frequencies and damping ratios of the plate. An identification approach is proposed based on the solution presented by $\mathrm{Li}$ et al. and by using the measured modal properties (i.e. natural frequencies and damping ratios). ${ }^{6}$ The proposed method is verified by using simulated and experimental results. The next section considers the free vibration analysis of an elastically supported plate.

\section{PLATE DYNAMICS ON ELASTIC SUPPORT ${ }^{6}$}

Figure 1 shows an elastically supported rectangular plate, which is constrained by lateral and torsional springs. It is considered that the elastic boundary condition contains structural damping.

The governing differential equation for the free vibration of the rectangular plate is expressed in Eq. (1):

$$
D \nabla^{4} w(x, y)-\rho h \omega^{2} w(x, y)=0 ;
$$

where $\nabla^{4}=\partial^{4} / \partial x^{4}+2 \partial^{4} / \partial x^{2} \partial y^{2}+\partial^{4} / \partial y^{4}$, and $w(x, y)$ is the lateral displacement function, $\omega$ is the angular frequency and $\rho, h$ and $D$ are mass density, thickness, and bending rigidity of the plate, respectively. The above governing equation is 